www.jmscr.igmpublication.org

Index Copernicus Value: 79.54

ISSN (e)-2347-176x ISSN (p) 2455-0450

crossrefDOI: https://dx.doi.org/10.18535/jmscr/v7i3.81

\title{
Comparison of Dosimetric Parameters in CT and MRI based planning in Image Guided Cervical Cancer Brachytherapy- Prospective Single Institutional Study
}

\author{
Authors \\ Saravanan.S ${ }^{1}$, Vijayasree T.N. ${ }^{2}$ \\ ${ }^{1}$ Professor Government Royapettah Hospital \& Kilpak Medical College, Chennai Tamil Nadu, India \\ ${ }^{2}$ Associate Professor, Govt Royapettah Hospital \& Kilpak Medical College, Chennai, Tamil Nadu, India \\ *Corresponding Author \\ Vijayasree $\mathbf{T} \mathbf{N}$ \\ Email: tnvijayasree@gmail.com
}

\begin{abstract}
Purpose: To compare the contours and dose-volume histograms (DVH) of the tumor and organs at risk $(O A R)$ with computed tomography (CT) vs. magnetic resonance imaging (MRI) in cervical cancer brachytherapy - prospective single institutional study.

Materials \& Methods: A total of 79 histologically proven cervical cancer patients of Stage IIB to IIIB, completed concurrent Chemoradiation were enrolled in a prospective Institutional Board approved brachytherapy protocol between March 2017 and May 2018. All of them underwent brachytherapy using a MRI-compatible tandom and ovoids applicator. Planning is done using both CT and MRI for the first fraction. The tumour and organs at risk (bladder, rectum and sigmoid) were contoured separately on CT and on MRI using clinical findings combined with GEC-ESTRO guidelines. The Dose Volume (DVH) parameters of Tumor and OARs were analysed using paired t test. $P$ values $<0.05$ were considered significant.

As per our Departmental protocol, all patients received $7 G y \times 3$ fractions.

Results: The mean V100 is higher for CT based planning compared to MRI and D90 is higher for MRI based planning with statistically significant difference ( $p$-). D2cc of bladder, rectum is same in both. D2cc for sigmoid is higher in MRI based planning.

Conclusion: Both CT and MRI based planning can be done. MRI gives better tissue delineation hence of HRCTV, resulting in lesser V100 and higher D90, so dose to actual tumor can be escalated in bulky disease with respect bladder and rectum.

Keywords: Carcinoma cervix, Brachytherapy, Computed tomography, Magnetic resonance imaging, Dosimetric Parameters.
\end{abstract}

\section{Introduction}

Cervical cancer ranks as fourth, in the incidence and mortality of cancer worldwide ${ }^{[1]}$. In India, among females, it is second, with an incidence of
$16.5 \%$ in 2018.Standard of care in locally advanced cervical cancer stage IIB to IVA is concurrent chemoradiation. Radiotherapy involves External Beam Radiotherapy followed by 
brachytherapy ${ }^{[2],[3]}$. Over the past 10years, World is moving from 2D based treatment planning in brachytherapy to 3 Dimensional Image-guided Brachytherapy (IGBT) based on Group Européen de Curiethérapie-European Society for Therapeutic Radiology and Oncology GECESTRO guidelines, ${ }^{[4],[5]}$. Compared to film based 2D planning, IGBT with CT based planning which provided visualization of applicators along with patient's anatomy and hence the doses to the clinical target volume (CTV) and Organs at risk (OAR) are known accurately ${ }^{[6],[7]}$. In 2005, Kristis et al, published a study on MRI based planning and reported the systematic development from CT to MRI based BT planning ${ }^{[8]}$. Towards the end of last decade Guidelines for MR based treatment planning gained importance ${ }^{[9]}$. This guideline recommends the use of $\mathrm{T} 2$ weighted $3 \mathrm{D}$ isotropic MR sequence to be used for discrimination of cervix and grey zones which facilitate target contouring. Clinical results with treatment based on MR images showed improved disease control and lesser side effects ${ }^{[10],[11]}$.

There are few studies in the literature comparing the efficacy of both. A prior study of 10 patients from Viswanathan et al. demonstrated that CTbased contours tended to overestimate tumor width, leading to significant differences in target coverage $^{[12]}$. In 2014, Comparison and consensus guidelines for delineation of clinical target volume for CT and MR-based brachytherapy in locally advanced cervical cancer have been published by the Radiation Therapy Oncology Group (RTOG) Gynecologic Cancer Working Group ${ }^{[13]}$.

MRI based IGBT is considered a Gold standard presently. In a Government set up like ours, obtaining MRI at time of Brachytherapy is logistically difficult and time-consuming. In contrast, CT is readily available and can be done in the Radiotherapy department itself and is less costly.

Hence we in this study compared DVH parameters of CT and MR based Treatment plans and analyzed the superiority of MRI vs. CT based treatment planning.

\section{Subjects and Methods}

In this, Institutional ethical committee approved Prospective Study done from March 2017 to May 2018, Patients with Histopathologically proven squamous carcinoma or adenosquamous carcinoma of the uterine cervix, FIGO IB2 to III $\mathrm{B}$, with age between 18yrs and $60 \mathrm{yrs}$ and completed EBRT 50Gy with concurrent Chemotherapy and referred for Brachytherapy, to our Department of Radiotherapy, were included in our study. Patients with metastatic and recurrent disease, patients having contraindication for MRI imaging were excluded.

Pre-implant evaluation and anesthetic assessment were done. In pre-implant clinical evaluation, clinical examination was done and local and parametrial extent of the disease at the time of brachytherapy was mapped. Under spinal anaesthesia, the patient was positioned in lithotomy. Bladder catheterized, the bulb filled with contrast and uterine length and vaginal roominess assessed to select the proper length of tandem and ovoids. Then CT/MR compatible applicator is placed, initially the tandem followed by vaginal ovoids. Vaginal packing is done and the rectal tube placed. CT Simulation was carried out using the SOMATOM Definition AS 20 widebore open model Siemens Simulator available in our department, which could take images of patients lying with applicators in place, with slice interval of $2 \mathrm{~mm}$. MRI was done using Siemens Magnetron Avanto Tim 1.5 tesla, available in the Radiodiagnosis department. The imaging was done with the applicator in place and included T2 FSE in the para-axial, para-sagittal and paracoronal planes. For both CT and MRI, images were taken from the level above the uterine fundus to the inferior border of the symphysis pubis and till any vaginal tumour extension on axial slices and transferred to treatment planning system (TPS). For the first fraction, both CT and MRI is done for each patient and planning done. Treatment planning was done using Oncentra TPS Version 4 and treatment carried out by Nucletron - micro Selectron - 18 channels HDR 
Brachytherapy Unit (Micros electron HDRV3, Nucletron, supplied by Elekta). First CT images were imported in TPS and HR-CTV and OARs were contoured. Both the clinical and imaging information was used while contouring HRCTV. Entire cervix along with parametrial and vaginal extension at the time of brachytherapy was taken as HRCTV, as per GECESTRO guidelines. For bladder, rectum and sigmoid, the outer wall is contoured. Fig.1 Catheter reconstruction was done and standard loading pattern as per our institutional protocol was done and dose optimization to point $\mathrm{A}$ was done with a prescription dose of $7 \mathrm{~Gy}$. The same procedure was repeated on MRI images. Fig.2

Treatment Schedule planned for Brachytherapy is 7Gy x 3fractions High Dose Rate (HDR) Brachytherapy using Adaptive Image Guidance, First fraction using MRI Guidance (fig.1) as well as CT Simulation (fig.2) and II and IIIrd Fractions using CT Simulation. Patients are treated with Dose optimization to Point A and also applying the High-Risk CTV concept (HR CTVD90) and Dose-Volume Constraints for OAR. The plan evaluation is done to keep the Dose Volume Constraints (D2cc) were EQD2 70-75Gy for rectum and sigmoid and 90 Gy for bladder $(\alpha / \beta$ values for a tumour being $10 \mathrm{~Gy}$ and $3 \mathrm{~Gy}$ for Late reacting Normal Tissue). Whenever possible, respecting Dose Volume Constraints for organs at risk, Dose to HRCTV achieved to > 85Gy EQD2 in Large volume tumors.

The dose received by at least $90 \%$ of the volume (D90) and the minimal target dose (D100), as well as percentage of volume receiving $100 \%$ (V100) or more than the prescribed dose were calculated using cumulative dose-volume histograms (DVHs) of the CT (HR-CTVCT) and MRI (HRCTVMRI). DVHs were evaluated for the dose to 2 $\mathrm{cm} 3$ for the bladder, rectum and sigmoid. D90 and V100 HRCTV, D2cc of OARs for CT and MRI based planning are reported in the dose/HDR fraction. The Dose Volume (DVH) parameters of Tumor and OARs were analyzed using the paired t-test. $\mathrm{P}$ values $<0.05$ were considered significant.

\section{Results}

From March2017 to May2018, 80 patients were enrolled, after getting concurrence from the Institutional ethical committee and proper consent from the patients. Among them, 79 completed the treatment as per the protocol. All patients completed 50Gy EBRT with concurrent weekly cisplatin, followed by 3 fractions of HDR Brachytherapy of $7 \mathrm{~Gy}$ each, taking into account the total treatment duration to be 8 weeks from the starting of EBRT.

The patients were of age between 30-70years, among which 29 (36\%) of them were between 41 and 50years and $26(32 \%)$ in 51 to 60 years. Stage of the disease is from IIA to IVB, among them $41(51.8 \%)$ patients were of FIGO Stage IIIB, $24(30.3 \%)$ in IIB. The total dose given to point $\mathrm{A}$ is $85.7 \mathrm{~Gy} \mathrm{EQD} 2$ as per the equation, $(\mathrm{EQD} 2=\mathrm{D}[(\mathrm{d}+\alpha / \beta) /(2+\alpha / \beta)]$, where $\mathrm{D}$ - total dose, $\mathrm{d}-\mathrm{dose} /$ fraction and $\alpha / \beta-3$ for the latereacting normal tissues and 10 - for the tumor. The Statistical analysis of DVH parameters is given in Table.1. The mean value of D90 for CT is 7.95 and MRI being 9.61 depicting higher Mean value for MRI compared to CT and Mean V100 is higher in CT. Paired sample ' $t$ ' test showed a significant difference in ' $\mathrm{P}$ ' value. There was no statistical difference in D2cc of bladder and rectum, though a significant $\mathrm{p}$-value was observed in D2cc Sigmoid. The correlation Graph for HRCTV D90 and V100 is given in Fig. 3 and 4.

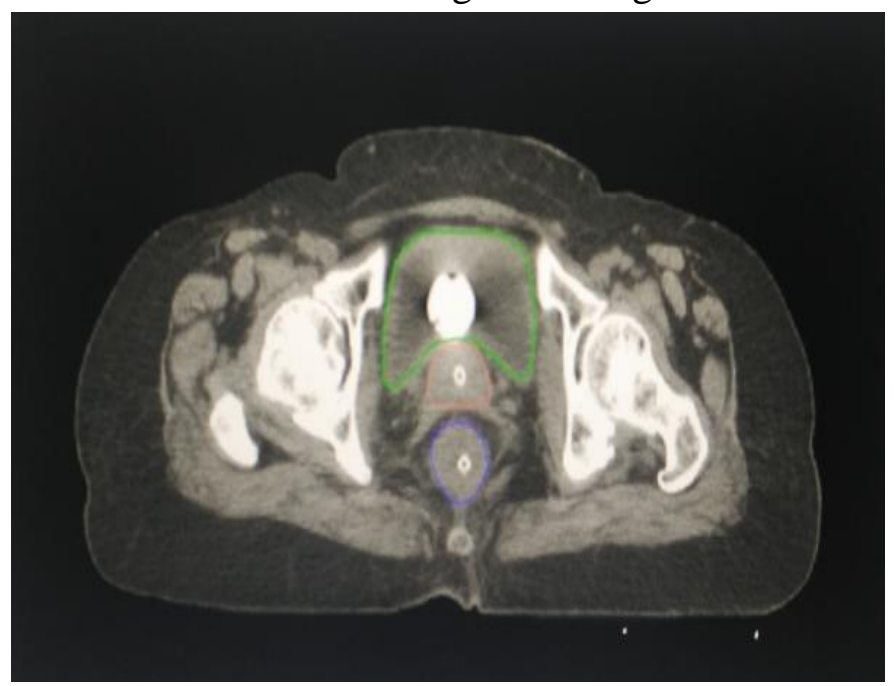

Figure 1 Contouring of HR-CTV and Bladder and rectum in $\mathrm{CT}$ images 


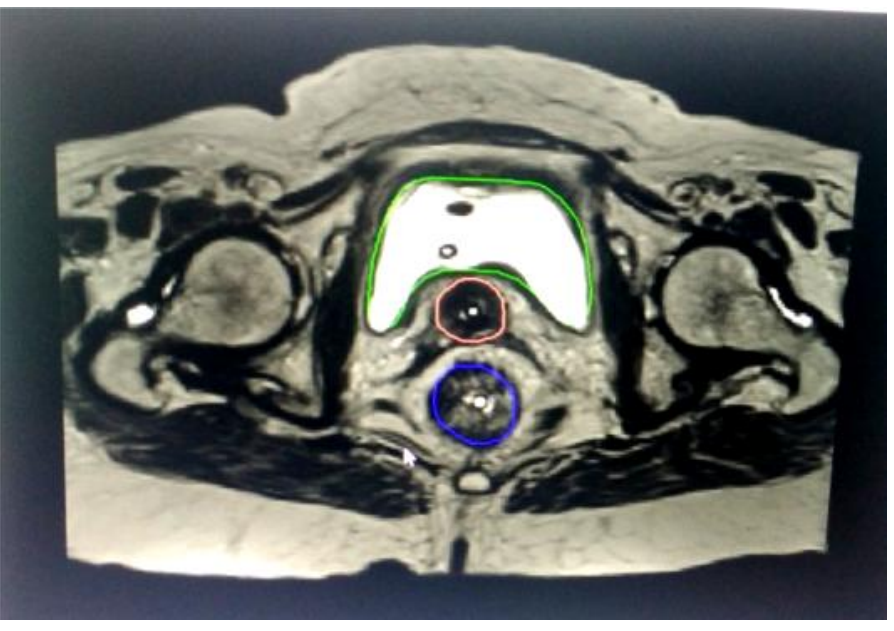

Figure 1.Contouring of HR-CTV and Bladder and rectum in MRI images

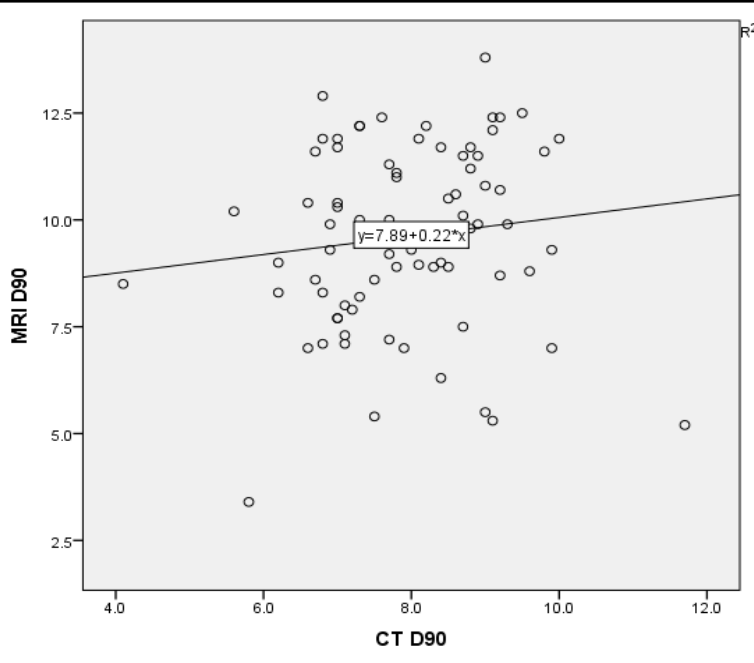

Fig.3 Correlation between MRI and CT findings D90

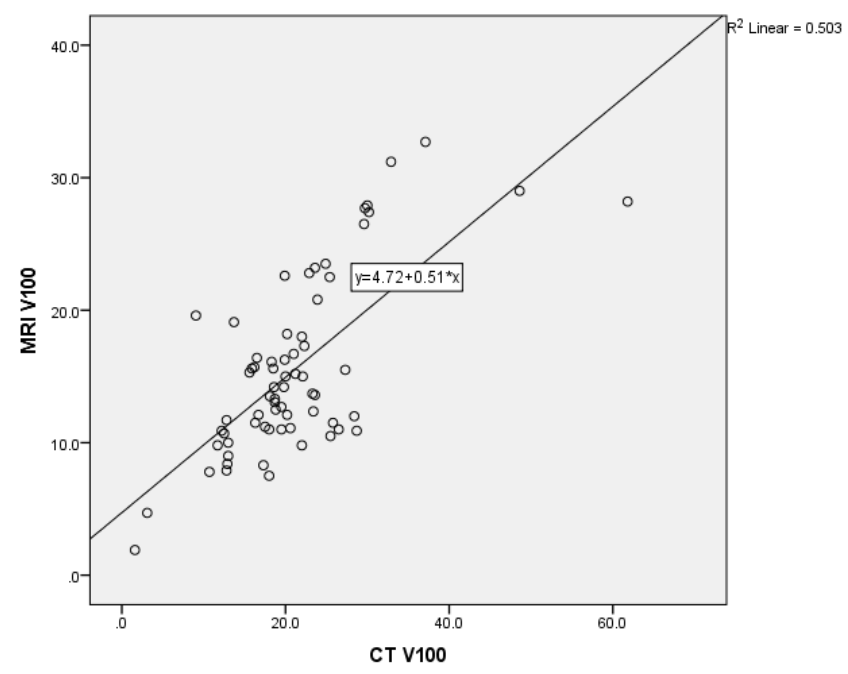

Fig.4 Correlation between MRI and CT findings - V100

Table 1 DVH Analysis

\begin{tabular}{|c|c|c|c|c|c|c|}
\hline Variable & Imaging method (n) & Range & Mean & Std.dev. & $\mathrm{t}$ value & p-value \\
\hline \multirow{2}{*}{ D 90} & CT (77) & $4.1-11.7$ & 7.95 & 1.22 & \multirow{2}{*}{-6.270} & \multirow{2}{*}{$<0.001 * * *$} \\
\hline & MRI (77) & $3.4-13.8$ & 9.61 & 2.14 & & \\
\hline \multirow{2}{*}{ V 100} & CT (68) & $1.6-61.8$ & 21.17 & 9.13 & \multirow{2}{*}{7.212} & \multirow{2}{*}{$<0.001 * * *$} \\
\hline & MRI (68) & $1.9-32.7$ & 15.54 & 6.58 & & \\
\hline \multirow{2}{*}{ Bladder D2cc } & CT (77) & $4.0-8.6$ & 6.50 & 1.06 & \multirow{2}{*}{1.270} & \multirow{2}{*}{0.208} \\
\hline & MRI (77) & $3.6-10.0$ & 6.33 & 1.18 & & \\
\hline \multirow{2}{*}{$\begin{array}{l}\text { Rectum } \\
\text { D2cc }\end{array}$} & CT (76) & $2.0-4.9$ & 4.01 & 0.67 & \multirow{2}{*}{0.998} & \multirow{2}{*}{0.322} \\
\hline & MRI (76) & $2.2-5.7$ & 3.90 & 0.76 & & \\
\hline \multirow{2}{*}{$\begin{array}{l}\text { Sigmoid colon } \\
\text { D2cc }\end{array}$} & CT (76) & $1.0-5.4$ & 2.65 & 1.08 & \multirow{2}{*}{-2.796} & \multirow{2}{*}{$0.007 * *$} \\
\hline & MRI (76) & $0.8-6.1$ & 2.97 & 1.16 & & \\
\hline
\end{tabular}

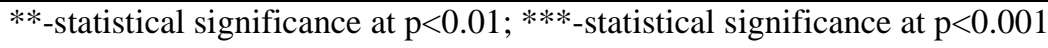

\section{Discussion}

In our prospective study with 79 patients, who underwent $\mathrm{CT}$ and MR based BT planning for Ist fraction and CT planning for II and III fractions, due to logistic reasons, we found CT overestimated HRCTV volume and D90 was lower. Reason being the parametrial involvement and endocervical involvement visualized clearly 
in MRI compared to CT. This correlates with, Consensus Guidelines by Viswanathan et al. in $2014^{[13]}$, saying mean tumor volume was smaller on MR than on CT $(\mathrm{P}<.001)$. A study by Wang et al. ${ }^{[14]}$ correlates with our study. Another study by Swanick et al. ${ }^{[15]}$, in 37 patients found that there is a discrepancy in CT and MR HRCTV in larger tumors and those with Parametrial involvement and that increases with increase in Body Mass Index. The explanation given for higher volumes shown in $\mathrm{CT}$ is that the nontumoral inflammation and scarring post EBRT in CT is not clearly seen apart from the residual tumor ${ }^{[16]}$. All these evidences are in favour that CT is inferior compared to MRI in BT planning.

Coming to OARs, for Bladder and Rectum, though the shape varied a little, D2cc remained almost the same with no statistical difference. This is in accordance with other studies by Rahul Krishnatry, Eskander and Zolciak-Siwinska ${ }^{[17]}$, ${ }^{[18]}$, and ${ }^{[19]}$. But D2ce sigmoid was found to have a significant $p$-value of $<0.01$ in our study.

To conclude, most of our patients presented with bulky tumours and parametrial involvement, hence there was a significant difference in HRCTV volume in CT and MRI.

\section{Conclusion}

MRI based BT planning is superior to CT based planning. When adopting volume-based treatment planning, this leads to dose difference (D90). Hence dose escalation is possible in bulky tumors without affecting OAR tolerances. Hence MR compatible Applicators if available, at least for Ist fraction MR based IGBT to be done. In developing countries like ours, if it's not possible, at least pre EBRT and pre BT MRI imaging should be done and those can be fused or the information can be incorporated in CT based IGBT during planning to get optimum doses to HRCTV and hence the response.

Sources of support in the form of grants: Nil

\section{References}

1. Global cancer statistics 2018: GLOBOCAN estimates of incidence and mortality worldwide for 36 cancers in 185 countries Freddie Bray $\mathrm{BSc}, \mathrm{MSc}, \mathrm{PhD}$ Jacques Ferlay ME, Isabelle Soerjomataram MD, MSc, PhD, Rebecca L. Siegel MPH,Lindsey A. Torre MSPH, Ahmedin Jemal PhD, DVM First published: 12 September 2018,

2. Lanciano RM, Martz K, Coia LR, Hanks GE. Tumour and treatment factors improving outcome in stage III-B cervix cancer. Int $\mathbf{J}$ Radiat. Oncol Biol Phys 1991;20:95-100.

3. Petereit DG, Pearcey R. Literature analysis of high dose rate brachytherapy fractionation schedules in the treatment of cervical cancer: is there an optimal fractionation schedule? Int $\mathbf{J}$ Radiat Oncol Biol Phys 1999;43:359-66.

4. Haie-Meder C, Potter R, Van Limbergen E, Briot E, De Brabandere M, Dimopoulos J, et al. Recommendations from Gynaecological (GYN) GEC-ESTRO Working Group (I): concepts and terms in 3D image based 3D treatment planning in cervix cancer brachytherapy with emphasis on MRI assessment of GTV and CTV. Radiother Oncol. 2005;74(3):235-45.

5. Potter R, Haie-Meder C, Van Limbergen E, Barillot I, De Brabandere M, Dimopoulos J, et al. Recommendations from gynaecological (GYN) GEC ESTRO working group (II): concepts and terms in 3D image-based treatment planning in cervix cancer brachytherapy-3D dose volume parameters and aspects of 3D image-based anatomy, radiation physics, radiobiology. Radiother Oncol. 2006;78(1):67-77.

6. Brodman M, Friedman F, Jr, Dottino P, Janus C, Plaxe S, Cohen C. A comparative study of computerized tomography, magnetic resonance imaging, and clinical staging for the detection of early cervix cancer. GynecolOncol. 1990;36 (3):409-12. [PubMed].

7. Mayr NA, Tali ET, Yuh WT, Brown BP, Wen $\mathrm{BC}$, Buller RE, et al. Cervical cancer: 
application of MR imaging in radiation therapy. Radiology 1993;189

(2):6018. [PubMed].

8. Kirisits C, Potter R, Lang S, Dimopoulos J, Wachter-Gerstner N, Georg D. Dose and volume parameters for MRI-based treatment planning in intracavitary brachytherapy for cervical cancer. Int J RadiatOncolBiol Phys. 2005;62(3):901-11.

9. Dimopoulos JC, Petrow P, Tanderup K, Petric $\mathrm{P}$, Berger D, Kirisits C, Pedersen EM, van Limbergen E, Haie-Meder C, Pötter R. Recommendations from Gynaecological (GYN) GEC-ESTRO Working Group (IV): Basic principles and parameters for MR imaging within the frame of image based adaptive cervix cancer brachytherapy. Radiother Oncol. 2012 Apr;103(1):113-22. doi: 10.1016/j.radonc. 2011.12.024. Epub 2012 Jan 30.

10. Pötter R, Georg P, Dimopoulos JCA, Grimm $\mathrm{M}$, Berger $\mathrm{D}$, Nesvacil $\mathrm{N}$, et al. Clinical outcome of protocol based image (MRI) guided adaptive brachytherapy combined with 3D conformal radiotherapy with or without chemotherapy in patients with locally advanced cervical cancer. Radiotherapy and Oncology. 2011;100 (1):116-23.

11. Lindegaard JC, Fokdal LU, Nielsen SK, JuulChristensen J, Tanderup K. MRI-guided adaptive radiotherapy in locally advanced cervical cancer from a Nordic perspective. ActaOncol. 2013;52 (7):1510-9.

12. Viswanathan AN, Dimopoulos J, Kirisits C, Berger D, Potter R. Computed tomography versus magnetic resonance imaging-based contouring in cervical cancer brachytherapy: results of a prospective trial and preliminary guidelines for standardized contours. Int $\mathbf{J}$ RadiatOncolBiol Phys. 2007; 68 (2):491-8.

13. Viswanathan AN, Erickson B, Gaffney DK, Beriwal S, Bhatia SK, Lee Burnett O, 3rd, et al. Comparison and consensus guidelines for delineation of clinical target volume for CTand MR-based brachytherapy in locally advanced cervical cancer. Int J Radiat Oncol Biol Phys. 2014; 90(2):320-8.

14. Wang F1, Tang Q1, Lv G2, Zhao F1, Jiang X1, Zhu X1, Li X1, Yan S3. Comparison of computed tomography and magnetic resonance imaging in cervical cancer brachytherapy: A systematic review. Brachytherapy. 2017 Mar Apr;16 (2):353-365.

15. Swanick CW, Castle KO, Vedam S, et al. Comparison of Computed Tomography- and Magnetic Resonance Imaging-based Clinical Target Volume Contours at Brachytherapy for Cervical Cancer. Int J RadiatOncolBiol Phys. 2016;96(4):793-800.

16. Vick CW, Walsh JW, Wheelock JB, Brewer WH. CT of the normal and abnormal parametria in cervical cancer. AJR Am J Roentgenol. 1984;143(3):597-603.

17. Rahul Krishnatry Firuza D. Patel Paramjeet Singh Suresh C. Sharma Arun S. Oinam Arvind K. Shukla, CT or MRI for Image-based Brachytherapy in Cervical Cancer. Japanese Journal of Clinical Oncology, Volume 42, Issue 4, 1 April 2012, Pages 309-313

18. Eskander RN, Scanderbeg D, Saenz CC, Brown M, Yashar C. Comparison of computed tomography and magnetic resonance imaging in cervical cancer brachytherapy target and normal tissue contouring. Int J Gynecol Cancer 2010;20:47-53.

19. Zolciak-Siwinska A, Kowalczyk A, Sikorska K, Bijok M, Michalski W, Gruszczynska E. Comparison of computed tomography with magnetic resonance imaging for imaging-based clinical target volume contours in cervical cancer brachytherapy. Brachytherapy. $2018 \mathrm{Jul}$ - Aug;17(4):667-672. 